\title{
Clinical Predictors of Drug Response in Patients with Obsessive-Compulsive Disorder
}

\author{
Chan-Hyung Kim${ }^{1}$, Jae-Wook Jeong ${ }^{2}$, Eun Ju Kim², Yoon Shick Shin ${ }^{3}$, Ho Suk Suh ${ }^{4}$, Hong Shick Lee ${ }^{5}$, \\ Min-Seong $\mathrm{KoO}^{2}$ \\ Department of Psychiatry, 'Severance Mental Health Hospital, Yonsei University, Gwangju, ${ }^{2}$ Kwandong University College of Medicine, \\ Goyang, ${ }^{3}$ Gangnam Severance Hospital, Yonsei University College of Medicine, Seoul, ${ }^{4}$ Gangnam Cha University College of Medicine, \\ Seoul, ${ }^{5}$ Yongin Mental Health Hospital, Yongin, Korea
}

\begin{abstract}
Objective: The aim of this study was to evaluate which clinical variables might influence the antiobsessional responses to proserotonergic drugs in a sample of patients with obsessive-compulsive disorder (OCD).

Methods: Two hundred forty-nine patients with DSM-IV OCD under-gone mean 13-month treatments with selective serotonin reuptake inhibitors. According to the treatment response, defined as a reductions of the Yale-Brown Obsessive Compulsive Scale (Y-BOCS) total score $\geq 35 \%$, patients were divided into two groups.

Results: One hundred fourteen patients responded to the treatment and the other one hundred thirty five patients did not. Responders had a significant long duration of medication in YUMC OCD clinic, short total duration of past treatment in other institutes, and higher frequency of drug naïve cases and lower baseline $Y$-BOCS scores.

Conclusion: The pre-treatment factors including total duration of past treatment, drug naïve or not, baseline OCD symptoms and the factor of duration of the treatment may influence drug treatment response in OCD patients.
\end{abstract}

KEY WORDS: Obsessive-compulsive disorder; Clinical predictors; Drug response; Treatment duration.

\section{INTRODUCTION}

Obsessive-compulsive disorder (OCD) is a mental disorder consisting of repetitive thoughts and compulsions. Recently, selective serotonin reuptake inhibitors (SSRI) have been used to improve the prognosis for OCD. However, $40-60 \%$ of OCD patients do not respond to SSRIs. ${ }^{1-4)}$ Research about clinical predictors, including clinical and demographic factors, has been conducted to develop treatment guidelines and predict the prognoses of OCD patients who show resistance to this kind of treatment. Several of the major prognostic factors were age at onset, ${ }^{5)}$ presence of tics, ${ }^{6,7)}$ social phobia, ${ }^{8)}$ personality disorders, ${ }^{9-11)}$ hoarding, ${ }^{12,13)}$ other types of OCD, ${ }^{14)}$ initial severity of OCD symptoms. ${ }^{3)}$ family history of $\mathrm{OCD},{ }^{15)}$ insight, ${ }^{16)}$ sex, ${ }^{17-19)}$ and others. A recent paper ${ }^{20)}$ showed the anti-obsessional and clinically predictive ef-

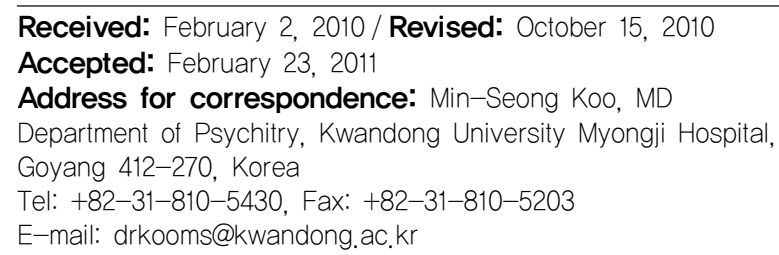

fects of sertraline, ${ }^{21)}$ but no studies have satisfactorily identified clinical predictors. Additionally, these studies addressed only a few partial factors and did not adequately identify the effects of various clinical factors on treatment response. Analyses of the effects of clinical and sociodemographic factors have suggested that information about clinical factors collected during initial patient evaluations can predict treatment responses and prognoses. We conducted a retrospective chart review study to investigate clinical predictors of responses to treatment with SSRIs according to sociodemographic and clinical factors.

\section{METHODS}

\section{Subjects}

This study included 249 patients with OCD who visited the YUMC (Yonsei University Medical Center) OCD clinic from October 1997 to October 2002. The subjects included participants $16-55$ years of age who were diagnosed with OCD according to the criteria in the Diagnostic and Statistical Manual of Mental Disorders, Fourth Edition (DSM-IV), and who provided informed consent 
Table 1. Characteristics of the total sample

\begin{tabular}{ll}
\hline & \multicolumn{1}{c}{ Total $(\mathrm{n}=246)$} \\
\hline Sex & Male $(\mathrm{n}=180)$, Female $(\mathrm{n}=66)$ \\
Age & $30.78(\mathrm{SD}=11.53)$ \\
Onset age & $21.42(\mathrm{SD}=9.69)$ \\
Duration of illness (months) & $102.21(\mathrm{SD}=82.05)$ \\
Comorbidity & \\
$\quad$ Mood disorder & $65(26.1 \%)$ \\
Personality disorder & $61(24.5 \%)$ \\
Anxiety disorder & $39(15.7 \%)$ \\
Tic disorder & $18(7.2 \%)$ \\
Somatization disorder & $3(1.2 \%)$ \\
Family History of OCD & $30(12.0 \%)$ \\
Family History of Tic disorder & $10(4.0 \%)$ \\
Poor insight cases & $111(44.6 \%)$ \\
Y-BOCS total baseline score & $29.22(\mathrm{SD}=6.93)$ \\
DOM (months) & $25.09(\mathrm{SD}=23.53)$ \\
DOP(months) & $18.15(\mathrm{SD}=18.61)$ \\
Drug naive cases & $90(36.1 \%)$ \\
\hline
\end{tabular}

n, number; R, Response; N, Non-response; Y-BOCS, Yale-Brown Obsessive-Compulsive Scale; OCD, Obsessive Compulsive Disorder; DOM, Duration of medication in YUMC OCD clinic; DOP, Duration of past treatment in other institutes.

for participation in this study. Exclusion criteria were 1) women who did not use contraception and had some possibility of being pregnant; 2) women who were pregnant or breast feeding; 3) those with a current or previous organic, neurological, or substance use disorder; and 4) patients with abnormal $\mathrm{CBC}$ values, urine analysis, liver function test, electrolytes, and EKGs.

\section{Evaluation of Clinical Manifestations}

The Yale-Brown Obsessive-Compulsive Scale (YBOCS $)^{22)}$ has been used to evaluate the severity of symptoms of patients with OCD. All patients were observed for 3-72 months, with an average observation period of 12.9 months $(\mathrm{SD}=13.8)$.

\section{Pharmacotherapy}

OCD symptoms were treated with drugs such as fluoxetine (40-80 mg/day), sertraline (100-150 mg/day), paroxetine (40-60 mg/day), and clomipramine (150-300 mg/ day). Benzodiazepines (alprazolam, clonazepam, lorazepam), mirtazapine, and trazodone were added if necessary for treating the OCD symptoms and comorbid disorders of each patient.

\section{Statistical Analysis}

Sociodemographic parameters and clinical variables were analyzed according to sex and previous responses to treatment. Continuous variables were analyzed with Student's t-test, and nominal variables were analyzed with chi-square tests. Participants were categorized into two groups according to clinical response. Those demonstrating at least a $35 \%$ improvement in scores on the Y-BOCS were placed into the responder group, and others were placed into the non-responder group. Groups were analyzed according to sociodemographic and clinical variables. Logistic regression analysis was performed to investigate the clinical predictors of drug response. All statistical analyses were conducted using SPSS version 10.0 (SPSS Inc., Chicago, NJ, USA) for Windows.

\section{RESULTS}

\section{Sociodemographic and Clinical Characteristics of Subjects (Table 1)}

The total sample consisted of 249 participants, including 180 males (72\%) and 66 females $(27 \%)$. The average age of subjects was $30.78(\mathrm{SD}=11.53)$ years, the average age of onset of OCD was $21.42(\mathrm{SD}=9.69)$ years, and the average duration of OCD was $102.21(\mathrm{SD}=82.05)$ months. The average total score on the total Y-BOS was 29.22 $(\mathrm{SD}=6.93) ; 65(26.1 \%)$ participants suffered from a mood disorder, $61(24.5 \%)$ met criteria for a personality disorder, $39(15.7 \%)$ had anxiety disorders, $18(7.2 \%)$ had tic disorders, and $3(1.2 \%)$ suffered from somatoform disorder. Additionally, 30 (12.0\%) reported a family history of OCD, and $10(4.0 \%)$ had a family history of tic disorders.

\section{Differences Between Responder and Non-Responder Groups in Terms of Clinical Parameters and Sociodemographic Factors (Table 2)}

The comparison of the sociodemographic and clinical characteristics of responders and non-responders revealed significant differences in baseline Y-BOS scores $(\mathrm{t}=2.245$, $\mathrm{df}=164, p=0.016$ ), duration of receiving medication at the YUMC OCD clinic $(\mathrm{t}=3.262, \mathrm{df}=163, p=0.001)$, duration of previous treatment at other institutions $(\mathrm{t}=2.044$, $\mathrm{df}=151, p=0.043)$, and initial drug-naïve status $\left(\lambda^{2}=\right.$ $6.538, \mathrm{df}=1, p=0.001$ ). However, no significant differences between responders and non-responders were observed in terms of sex, age, age at onset, duration of illness, comorbid disorders, family history of OCD, family history of tic disorders, and level of insight.

\section{Clinical Subtypes and Comorbid Disorders of Patients with OCD (Table 3)}

As noted above, 65 participants had comorbid mood disorders (26.1\%) such as depression, 39 had anxiety dis- 
Table 2. Characteristics of the sample according to clinical response

\begin{tabular}{|c|c|c|c|c|}
\hline & $R(n=114)$ & $N(n=135)$ & \multicolumn{2}{|c|}{ Statistics } \\
\hline Sex & $M(78), F(36)$ & $M(102), F(33)$ & $\lambda^{2}=0.959$ & $p=0.327$ \\
\hline Age & $31.46(12.86)$ & $30.21(10.36)$ & $\mathrm{T}=0.689$ & $p=0.492$ \\
\hline Onset age & $22.53(11.23)$ & $20.47(8.09)$ & $\mathrm{T}=1.361$ & $p=0.175$ \\
\hline Duration of illness (months) & $102.48(78.78)$ & $101.98(85.19)$ & $\mathrm{T}=0.040$ & $p=0.968$ \\
\hline \multicolumn{5}{|l|}{ Comorbidity } \\
\hline Mood disorder & 24 & 41 & $\lambda^{2}=2.771$ & $p=0.096$ \\
\hline Personality disorder & 24 & 37 & $\lambda^{2}=1.344$ & $p=0.246$ \\
\hline Anxiety disorder & 21 & 18 & $\lambda^{2}=1.206$ & $p=0.272$ \\
\hline Tic disorder & 7 & 11 & $\lambda^{2}=0.370$ & $p=0.543$ \\
\hline Somatization disorder & 1 & 2 & $\lambda^{2}=0.189$ & $p=0.664$ \\
\hline Family History of OCD & 10 & 20 & $\lambda^{2}=2.121$ & $p=0.145$ \\
\hline Family History of Tic disorder & 4 & 6 & $\lambda^{2}=0.140$ & $p=0.708$ \\
\hline Poor insight cases & 45 & 66 & $\lambda^{2}=2.209$ & $p=0.137$ \\
\hline Y-BOCS total baseline score* & $27.94(7.18)$ & $30.31(5.21)$ & $\mathrm{T}=2.245$ & $p=0.016$ \\
\hline $\mathrm{DOM}^{\dagger}$ (months) & $31.86(24.66)$ & $19.38(22.59)$ & $T=3.262$ & $p=0.001$ \\
\hline $\mathrm{DOP}^{*}$ (months) & $14.19(15.44)$ & $21.52(21.30)$ & $\mathrm{T}=2.044$ & $p=0.043$ \\
\hline Drug naive cases $^{\dagger}$ & 54 & 36 & $\lambda^{2}=6.538$ & $p=0.001$ \\
\hline
\end{tabular}

R: Response, N: Non-response, n: number, M: male, F: female, Y-BOCS: Yale-Brown Obsessive-Compulsive Scale, OCD: Obsessive Compulsive Disorder, DOM: Duration of medication in YUMC OCD clinic, DOP: Duration of past treatment in other institutes.

${ }^{*} p<0.05,{ }^{+} p<0.001$.

Table 3. Comorbidity of obsessive compulsive disorder

\begin{tabular}{|c|c|c|c|c|}
\hline Disease entities & Specific diseases & $\mathrm{T}(\%)$ & R (\%) & $\mathrm{N}(\%)$ \\
\hline \multirow[t]{4}{*}{ Mood disorder $(n=65)$} & Depression & $57(22.9)$ & $21(18.4)$ & $36(26.7)$ \\
\hline & Dysthymia & $3(1.2)$ & $2(1.8)$ & $1(0.7)$ \\
\hline & Bipolar disorder & $3(1.2)$ & $1(0.9)$ & $2(1.5)$ \\
\hline & Other emotional problem & $2(8)$ & 0 & $2(1.5)$ \\
\hline \multirow[t]{5}{*}{ Anxiety disorder $(n=39)$} & Panic disorder & $7(2.8)$ & $4(3.5)$ & $3(2.2)$ \\
\hline & Hypochondriasis & $6(2.4)$ & $3(2.6)$ & $3(2.2)$ \\
\hline & Social phobia & $15(6)$ & $9(7.8)$ & $6(4.4)$ \\
\hline & GAD & $3(1.2)$ & $3(2.6)$ & 0 \\
\hline & Other anxiety disorder & $8(3.2)$ & $2(1.8)$ & $6(4.4)$ \\
\hline Somatization disorder $(n=3)$ & Somatization disorder & $3(1.2)$ & $1(0.9)$ & $2(1.5)$ \\
\hline \multirow[t]{7}{*}{ Personality disorder $(n=61)$} & Obsessive compulsive PD & $30(12)$ & $10(8.8)$ & $20(14.8)$ \\
\hline & Avoidant PD & $15(6)$ & $9(7.9)$ & $6(4.4)$ \\
\hline & Schizoid PD & $3(1.2)$ & $1(0.9)$ & $2(1.5)$ \\
\hline & Borderline PD & $5(2)$ & $1(0.9)$ & $4(3)$ \\
\hline & Histrionic PD & $3(1.2)$ & $1(0.9)$ & $2(1.5)$ \\
\hline & Antisocial PD & $2(0.8)$ & $1(0.9)$ & $1(0.7)$ \\
\hline & Schizotypal PD & $3(1.2)$ & $1(0.9)$ & $2(1.5)$ \\
\hline \multirow[t]{2}{*}{ Child onset diseases $(n=22)$} & ADHD & $4(1.6)$ & $1(0.9)$ & $3(2.2)$ \\
\hline & Tic disorder & $18(7.2)$ & $7(6.1)$ & $11(8.1)$ \\
\hline Psychotic disorder $(n=15)$ & Psychotic disorder NOS & $15(6)$ & $6(5.2)$ & $9(6.7)$ \\
\hline \multirow[t]{2}{*}{ Others $(n=4)$} & Alcohol dependence & $3(1.2)$ & $1(0.9)$ & $2(1.5)$ \\
\hline & PMS & $1(0.4)$ & 0 & $1(0.7)$ \\
\hline
\end{tabular}

T: Total, R: Responder, N: Non-responder, n: number, \%: Percent, GAD: Generalized anxiety disorder, PD: Personality disorder, ADHD: Attention deficit hyperactivities disorder, NOS: Not otherwise specific, PMS: Pre-menstrual syndrome some patients have more than 2 specific diseases.

orders (15.6\%), 61 had personality disorders $(24.5 \%)$, and 18 had tic disorders (7.2\%). No significant difference between responders and non-responders were observed in terms of comorbid disorders. Twenty-four responders met criteria for mood disorders (21.0\%), 24 for personality disorders $(21.0 \%), 21$ for anxiety disorders (18.4\%), and one for somatoform disorder $(0.9 \%)$. The non-responders group included 41 participants with mood disorders (30.3\%), 37 with personality disorders (27.4\%), 12 with anxiety disorders $(9 \%)$, and two with somatoform disorder $(1.5 \%)$. 
Table 4. Subtypes of Obsessive symptoms according to clinical response

\begin{tabular}{|c|c|c|c|}
\hline Types & $\mathrm{T}(\%)$ & $\mathrm{R}(\%)$ & N (\%) \\
\hline Checking type compulsion & $60(24.1)$ & $21(18.4)$ & $39(28.9)$ \\
\hline Washing type compulsion & $59(23.7)$ & $33(28.9)$ & $26(19.2)$ \\
\hline Pathologic doubt obsession & $28(11.2)$ & $12(10.5)$ & $16(11.9)$ \\
\hline Symmetry and precision type compulsion & $17(6.8)$ & $12(10.5)$ & $5(3.7)$ \\
\hline Aggression obsession & $15(6.0)$ & $7(6.1)$ & $8(5.9)$ \\
\hline Counting type compulsion & $13(5.2)$ & $4(3.5)$ & $9(6.7)$ \\
\hline Hoarding type compulsion & $12(4.8)$ & $6(5.2)$ & $6(4.4)$ \\
\hline Somatization obsession & $12(4.8)$ & $6(5.2)$ & $6(4.4)$ \\
\hline Need to ask or confess type compulsion & $11(4.4)$ & $5(4.3)$ & $6(4.4)$ \\
\hline Sexual obsession & $9(3.6)$ & $4(3.5)$ & $5(3.7)$ \\
\hline Need for symmetry obsession & $6(2.4)$ & $2(1.8)$ & $4(3)$ \\
\hline Contamination obsession & $3(1.2)$ & $1(0.9)$ & $2(1.5)$ \\
\hline Others & $4(1.6)$ & $2(1.8)$ & $2(1.5)$ \\
\hline Totals & 249 & 114 & 135 \\
\hline
\end{tabular}

R: Response, N: Non-response.

Chi-Square Tests ( $d f=19$, chi-square 19, $p=0.612$ ).

Table 5. Summary of logistic regression results*

\begin{tabular}{lccccc}
\hline \multicolumn{1}{c}{ Variable } & B & S.E. & Wald & df & Significance \\
\hline Drug-naïve & -0.991 & 0.442 & 5.033 & 1 & 0.025 \\
DOM & -0.092 & 0.018 & 26.210 & 1 & 0.000 \\
DOP & 0.091 & 0.021 & 18.575 & 1 & 0.371 \\
Y-BOCS & 0.026 & 0.030 & 0.729 & 1 & 0.912 \\
Constant & 0.695 & 0.974 & 0.508 & 1 & 0.393 \\
\hline
\end{tabular}

B: Bonferroni coefficient, S.E.: Standard Error, Wald: Wald statistics, df: degree of freedom, significance: significance of Wald test, Exp(B): exponential $\beta$ (odds ratio), DOM: Duration of medication in YUMC OCD clinic, DOP: Duration of past treatment in other institutes, Y-BOCS: Yale-Brown Obsessive-Compulsive Scale.

*Model Chi-square 51.530, $p=0.000$, Cox \& Snell $R^{2} 0.368$, Nagelkerke $R^{2} 0.458$.

\section{Differences Between Responders and Non-Responders according to Clinical Subtype of OCD (Table 4)}

The total sample of 249 patients included 60 (24.1\%) participants with the checking subtype of OCD, 59 (23.7\%) with the washing subtype, $28(11.2 \%)$ with the pathological doubt subtype, $17(6.8 \%)$ with the symmetry and precision subtype, and $15(6.0 \%)$ with the aggressive subtype. No significant differences between responders and non-responders were observed in terms of subtype. Among responders, 33 (28.9\%) had the washing subtype, $21(18.4 \%)$ had the checking subtype, $12(10.5 \%)$ had the pathological doubt subtype, and $12(10.5 \%)$ had the symmetry and precision subtype. The non-responders group included 39 (28.9\%) with the checking subtype, 26 $(19.2 \%)$ with the washing subtype, $16(11.9 \%)$ with the pathological doubt subtype, and $9(6.7 \%)$ with the counting subtype.

\section{Clinical Predictors of Drug Responses in OCD Patient According to the Logistic Regression Analysis (Table 5)}

The clinical predictors of drug responses were investigated using logistic regression to analyze the parameters that differentiated between responders and non-responders. Total baseline scores on the Y-BOCS, duration of receiving medication at the YUMC OCD clinic, duration of previous treatment at other institutions, and status as drug-naïve before the study were chosen as independent variables, and clinical symptomatology was selected as the dependent variable. The analysis showed that the duration of receiving medication at the YUMC OCD clinic was the most significant predictor of drug response and that the duration of previous treatment at other institutions, status as drug-naïve before the study, and total baseline scores on the Y-BOS were also significant clinical predictors (Table 5).

\section{DISCUSSION}

This study investigated the major clinical predictors of drug responses among OCD patient. For this purpose, we examined various parameters that potentially influence the treatment of OCD, including the sociodemographic characteristics and initial clinical status of patients visiting the YUMC OCD clinic. According to this study, the dura- 
tion of pharmacological treatment at the YUMC OCD clinic exerted the strongest influence on treatment response. Additionally, duration of treatment at other institutions, prior drug-naïve status, and baseline Y-BOS scores were also found to be significant predictors of response to medication (Table 2).

These results may be understood in terms of the time needed for medication, education, and other types of treatment to show effectiveness. According to studies conducted by Grimshaw ${ }^{23)}$ and Goodwin et al. ${ }^{24)}$ on the appropriate medication duration for OCD patients, most therapeutic effects appear within the first year. Thus, this study's use of 13 months as the average period of observation was consistent with the other reports. ${ }^{23-25)}$ However, additional longer-term research on the importance of medication duration is required because this study was limited to an average of only 13 months.

This study investigated predictors according to the clinical profiles obtained during initial visits to our facility. The duration of previous treatment at other institutions, previous drug-naïve status, and baseline Y-BOS scores were found to be important clinical predictors of medication response. Given that more responders than non-responders had been drug-naïve or had received medication only briefly in the past, we can hypothesize that inappropriate anti-obsessional pharmacotherapy may decrease the effect of treatment. That fewer responders initially reported severe symptoms and that the number of responders increased as the duration of pharmacological treatment increased indicated that longer periods of medication are required for patients with more severe obsessive-compulsive symptoms. This finding is consistent with the study conducted by de Haan et al., ${ }^{26)}$ which showed that OCD patient with severe symptoms needed more medication than did OCD patients with mild symptoms.

This study found no relationship between treatment response and sex. Because the ratio of male to female participants was 180:69, additional research with a more evenly divided sample is necessary. Additionally, no significant differences in treatment response related to comorbid psychopathology, including the presence of personality disorders, was observed in this study (Table 2). However, the finding of significant differences between responders and non-responders with respect to the presence of depression and obsessive-compulsive personality disorder requires further study.

No significant differences in treatment response according to clinical subtype of OCD was observed in this study; however, the washing subtype was the most prevalent subtype in the responder group, and the checking subtype was the most prevalent subtype in the non-responder group. No differences in treatment response was observed between those with the hoarding and those with the somatization subtypes, which has been suggested as significant distinction in terms of predicting treatment response in patients with OCD. ${ }^{27,28)}$ Additionally, no significant differences in clinical responses were observed according to level of insight or presence of family history of tic disorders.

Thus, the duration of receiving medication at the YUMC OCD clinic, the duration of previous treatment at other institutions, prior drug-naïve status, and baseline Y-BOS scores are the major clinical predictors of responses to medication among patients with OCD.

This study has the following limitations. First, no double-blind control group was used. Second, because this research was based on a natural follow-up of data obtained from patients who visited only the YUMC OCD clinic, these results may not be generalizable to all OCD patients. Third, patients received various types of medications. More accurate clinical results will require the use of a standardized treatment protocol. Indeed, a standardized medication regimen that is most effective for Korean patients with OCD needs to be developed by conducting comparison studies on treatment with various medications.

Finally, this study is limited by its inability to study long-term treatment effects because, although some patients were followed for 72 months, the average treatment duration was 13 months. Thus, a longer-term follow-up study among a consistent group of patients is required. Despite these restrictions, this study contributed to the literature by broadly examining the role of sociodemographic and clinical factors as predictors of the treatment response of patients with OCD and by suggesting areas warranting further research in the future.

\section{REFERENCES}

1. Pessina E, Albert U, Bogetto F, Maina G. Aripiprazole augmentation of serotonin reuptake inhibitors in treatment-resistant obsessive-compulsive disorder: a 12-week open-label preliminary study. Int Clin Psychopharmacol 2009;24:265-269.

2. Stein DJ. Obsessive-compulsive disorder. Lancet 2002;360: 397-405.

3. Alarcon RD, Libb JW, Spitler D. A predictive study of obsessive-compulsive disorder response to clomipramine. $J$ Clin Psychopharmacol 1993;13:210-213.

4. Ravizza L, Barzega G, Bellino S, Bogetto F, Maina G. 
Predictors of drug treatment response in obsessive-compulsive disorder. J Clin Psychiatry 1995;56:368-373.

5. Ackerman DL, Greenland S, Bystritsky A, Morgenstern H, Katz RJ. Predictors of treatment response in obsessive-compulsive disorder: multivariate analyses from a multicenter trial of clomipramine. J Clin Psychopharmacol 1994;14: 247-254.

6. McDougle CJ, Goodman WK, Leckman JF, Barr LC, Heninger GR, Price LH. The efficacy of fluvoxamine in obsessive-compulsive disorder: effects of comorbid chronic tic disorder. J Clin Psychopharmacol 1993;13:354-358.

7. McDougle CJ, Goodman WK, Leckman JF, Lee NC, Heninger GR, Price LH. Haloperidol addition in fluvoxamine-refractory obsessive-compulsive disorder. A doubleblind, placebo-controlled study in patients with and without tics. Arch Gen Psychiatry 1994;51:302-308.

8. Carrasco JL, Hollander E, Schneier FR, Liebowitz MR. Treatment outcome of obsessive compulsive disorder with comorbid social phobia. J Clin Psychiatry 1992;53:387-391.

9. Baer L, Jenike MA, Black DW, Treece C, Rosenfeld R, Greist J. Effect of axis II diagnoses on treatment outcome with clomipramine in 55 patients with obsessive-compulsive disorder. Arch Gen Psychiatry 1992;49:862-866.

10. Mundo E, Erzegovesi S, Bellodi L. Follow-up of obsessivecompulsive patients treated with proserotonergic agents. $J$ Clin Psychopharmacol 1995; 15:288-289.

11. Cavedini P, Erzegovesi S, Ronchi P, Bellodi L. Predictive value of obsessive-compulsive personality disorder in antiobsessional pharmacological treatment. Eur Neuropsychopharmacol 1997;7:45-49.

12. Black DW, Monahan P, Gable J, Blum N, Clancy G, Baker P. Hoarding and treatment response in 38 nondepressed subjects with obsessive-compulsive disorder. J Clin Psychiatry 1998;59:420-425.

13. Mataix-Cols D, Rauch SL, Manzo PA, Jenike MA, Baer L. Use of factor-analyzed symptom dimensions to predict outcome with serotonin reuptake inhibitors and placebo in the treatment of obsessive-compulsive disorder. Am J Psychiatry 1999;156:1409-1416.

14. Foa EB, Kozak MJ, Goodman WK, Hollander E, Jenike MA, Rasmussen SA. DSM-IV field trial: obsessive-compulsive disorder. Am J Psychiatry 1995;152:90-96.

15. Ronchi P, Abruzzese M, Erzegovesi S, Diaferia G, Sciuto G, Bellodi L. The epidemiology of obsessive-compulsive disorder in an Italian population. Eur Psychiatry 1992;7: 53-59.
16. Erzegovesi S, Cavallini MC, Cavedini P, Diaferia G, Locatelli M, Bellodi L. Clinical predictors of drug response in obsessive-compulsive disorder. J Clin Psychopharmacol 2001;21:488-492.

17. Rasmussen SA, Eisen JL. Epidemiology of obsessive compulsive disorder. J Clin Psychiatry 1990;51 Suppl:10-13.

18. Bellodi L, Sciuto G, Diaferia G, Ronchi P, Smeraldi E. Psychiatric disorders in the families of patients with obsessive-compulsive disorder. Psychiatry Res 1992;42:111-120.

19. Lensi P, Cassano GB, Correddu G, Ravagli S, Kunovac JL, Akiskal HS. Obsessive-compulsive disorder. Familial-developmental history, symptomatology, comorbidity and course with special reference to gender-related differences. $\mathrm{Br} J$ Psychiatry 1996;169:101-107.

20. Kim CH, Song DH, Lee HS, Choi NK. An open trial fluoxetine in the treatment of obsessive-compulsive disorder. Korean J Psychopharmacol 1995:6;100-104.

21. Yoo EJ, Woo HW, Kim YC, Yun KW, Kim JW, Lim WJ. A study of predictive factors of treatment response to sertraline in patients with obsessive-compulsive disorder. Korean J Neuropsychiatr Assoc 2000:39;435-444.

22. Goodman WK, Price LH, Rasmussen SA, Mazure C, Delgado P, Heninger GR, et al. The Yale-Brown Obsessive Compulsive Scale. II. Validity. Arch Gen Psychiatry 1989;46:1012-1016.

23. Grimshaw L. The outcome of obsessional disorder. A follow-up study of 100 cases. Br J Psychiatry 1965;111: 1051-1056.

24. Goodwin DW, Guze SB, Robins E. Follow-up studies in obsessional neurosis. Arch Gen Psychiatry 1969;20:182-187.

25. Skoog G, Skoog I. A 40-year follow-up of patients with obsessive-compulsive disorder. Arch Gen Psychiatry 1999; 56:121-127.

26. de Haan E, van Oppen P, van Balkom AJ, Spinhoven P, Hoogduin KA, Van Dyck R. Prediction of outcome and early vs. late improvement in OCD patients treated with cognitive behaviour therapy and pharmacotherapy. Acta Psychiatr Scand 1997;96:354-361.

27. Mataix-Cols D, Rauch SL, Baer L, Eisen JL, Shera DM, Goodman WK, et al. Symptom stability in adult obsessivecompulsive disorder: data from a naturalistic two-year follow-up study. Am J Psychiatry 2002;159:263-268.

28. Black DW, Monahan P, Gable J, Blum N, Clancy G, Baker P. Hoarding and treatment response in 38 nondepressed subjects with obsessive-compulsive disorder. J Clin Psychiatry 1998;59:420-425. 